\title{
Florecimiento algal nocivo de Pyrodinium bahamense en diciembre 2018 en la costa del Pacífico de Guatemala
}

\author{
Harmful algal bloom of Pyrodinium bahamense in December 2018 in the Pacific coast of \\ Guatemala \\ Josué García-Pérez¹, Alan García-Lopez², Leonel Carrillo-Ovalle, \\ Nicolás Solares-Cortez ${ }^{3}$, Rubén López-Bran ${ }^{4}$ \\ ${ }^{1}$ Centro de Estudios del Mar y Acuicultura, Universidad de San Carlos de Guatemala, \\ ${ }^{2}$ Instituto Nacional de Sismología, Vulcanología, Meteorología e Hidrología de Guatemala, \\ ${ }^{3}$ Departamento de Observación e Investigación Marítima, Empresa Portuaria Quetzal, \\ ${ }^{4}$ Dirección de Normatividad de Pesca y Acuicultura, Ministerio de Agricultura, Ganadería y Alimentación, Guatemala.
}

*Autor al que se dirige la correspondencia: josgar85@gmail.com

Recibido: 14 de agosto 2019 / Revisión: 20 de enero 2020 / Aceptado: 11 de febrero 2020

\section{Resumen}

$\mathrm{L}$ os florecimientos algales nocivos (FAN) son eventos naturales que ocurren cuando una o más especies de microalgas proliferan en concentraciones que pueden causar daño a los organismos acuáticos y a seres humanos que los consuman. En las últimas décadas, se ha registrado a nivel mundial un aumento de eventos de mortalidad alarmante de organismos acuáticos e intoxicaciones en seres humanos causadas por toxinas producidas por microalgas. En Guatemala existe escasa información sobre eventos FAN, no obstante, en diciembre de 2018 ocurrió un FAN en la costa Pacífico de Guatemala. La Comisión Nacional para la Vigilancia y el Control de la Marea Roja Tóxica recolectó muestras biológicas e identificó la presencia de 3,000 cel/L del dinoflagelado Pyrodinium bahamense. El bioensayo en ratón de las muestras, indica concentraciones de saxitoxina de $8,236 \mathrm{UR} / 100$ g y 6,559 UR/100 g, para los días 20 y 27 de diciembre, respectivamente. Estas concentraciones no han sido reportadas previamente en Guatemala, y pueden ser potencialmente tóxicas para la salud pública. Se recomienda mantener un monitoreo de FAN para prevenir impactos negativos en la salud pública y ambiental.

Palabras claves: Fitoplancton, dinoflagelados planctónicos, marea roja, saxitoxina

\begin{abstract}
$\mathrm{H}$ armful algal blooms (HABs) are natural events that occur when one or more species of microalgae proliferate at concentrations that can cause damage to aquatic organisms and to those who consume them. In Guatemala very little information exists on HAB events, although in December 2018 has occurred a HABs at the Pacific Coast of Guatemala. A biological sample were collected by the National Commission for Surveillance and Control of Toxic Red Tide. The samples showed 3,000 cel/L of the dinoflagellate Pyrodinium bahamense. During the bioassay for saxitoxin, the concentrations reached 8,236 MU/100 g and 6,559 MU/100 g, for December 20th and 27 th, respectively. These concentrations has not been reported previously for Guatemala, and could be a risk in the public health. This result emphasizes the importance of maintaining the HABS monitoring program to prevent negative impact on public environmental health.
\end{abstract}

Keywords: Phytoplankton, planktonic dinoflagellates, red tides, saxitoxin

| (c) (1) (2) La reproducción total o parcial del contenido e imágenes de esta publicación se rige de acuerdo a normas internacionales sobre protección a los derechos de autor, con criterio especificados en la licencia Creative Commons (CC BY-NC-SA 4.0) 


\section{Introducción}

El fitoplancton marino está conformado por una gran diversidad de microalgas, entre las que se encuentran aquellas que forman Florecimientos Algales Nocivos (FAN) o HABs en inglés (Harmful Algal Blooms), antes llamados mareas rojas, porque su presencia puede cambiar el color del agua superficial (Herrera-Sepúlveda, Sierra-Beltrán, \& Hernández-Saavedra, 2008). Los FAN consisten en un evento natural donde una o más especies de microalgas, usualmente dinoflagelados, diatomeas o cianobacterias, aumenta su abundancia y producen sustancias químicas (toxinas) en concentraciones que afectan a otros organismos en el medio acuático y al ser humano (Band-Schmidt, Bustillos-Guzmán, López-Cortez, Nunez-Vasquez, \& Hernández-Sandoval, 2011; Smayda, 1997).

En las últimas décadas, se ha registrado a nivel mundial un aumento de eventos FAN con consecuencias catastróficas para el hábitat acuático, incluyendo mortalidades masivas de organismos acuáticos silvestres y de cultivo (Van Dolah, 2000). Estos fenómenos también son motivo de preocupación para la salud pública, ya que inclusive el contacto con el agua o la inhalación de aerosoles marinos pueden provocar intoxicación en humanos, así como el consumo de organismos marinos afectados por las microalgas nocivas puede causar complicaciones clínicas e inclusive la muerte por parálisis (Berdalet et al., 2011), como en el caso de la intoxicación paralítica por mariscos (PSP: Paralytic shellfish poisoning; siglas en inglés), que ocurre por ingesta de moluscos bivalvos contaminados con toxinas, comúnmente llamadas saxitoxinas, que provocan parálisis musculares muy serias, hasta causar la muerte en los casos más severos (Herrera-Sepúlveda et al., 2008; Plate, 1906; Van Dolah, 2000; ).

En general, las intoxicaciones paralizantes por mariscos son provocadas por dinoflagelados de los géneros Alexandrium, Gymnodinium y Pyrodinium que habitan en aguas tropicales y templadas. En comunidades costeras del Pacífico, la especie que ha provocado más daños es Pyrodinium bahamense, que es un dinoflagelado tecado ampliamente distribuido en las costas del norte, centro y sur de América (Alonso-Rodríguez, Mendoza-Amezquita, Velásquez-López, Seim, \& Martínez-Rodríguez, 2015).

En Guatemala, el primer caso documentado de intoxicación por dinoflagelados fue provocado por el consumo de bivalvos de la especie Amphichaena kindermanni (Rosales-Loessener, 1989; Rosales- Loes- sener, De Porras, \& Dix, 1989) contaminados con $P$. bahamense variedad compressum en julio de 1987, afectó a 187 personas y causó la muerte de 26, por acumulación de saxitoxina. A partir de esa fecha se han registrado en Guatemala florecimientos de $P$. $b a$ hamense var. compressum en 1989, 1990, 1995, 2001, 2005, 2007 y 2009 (Carrillo-Ovalle, 2009), pero no se reportaron pérdidas humanas. Guatemala, por medio de este reporte, confirma nuevamente la presencia de $P$. bahamense, con concentraciones de saxitoxina de 8,236 UR/100 g y 6,559 UR/100 g, para los días 20 y 27 de diciembre de 2018; valores nunca antes reportados para Guatemala y Latinoamérica, y que pueden ser un riesgo potencial para la salud pública y ambiental.

\section{Presentación del Caso}

A inicios de diciembre de 2018, se observaron decoloraciones de formas irregulares a lo largo de la superficie del mar en Puerto Quetzal en la costa del Pacífico de Guatemala. La concentración media de clorofila (desviación estándar), registrada en imágenes del satélite MODIS-Aqua durante el mes, fue 18 (2) $\mathrm{mg} / \mathrm{m}^{3}$; superior a la media mensual 1.64 (11) $\mathrm{mg} / \mathrm{m}^{3}$ para el período de octubre 2018 a enero 2019 (Figura 1), que indicó la presencia de florecimientos algales, siendo atribuidos a diversos géneros de dinoflagelados y microalgas planctónicas.

El 10 de diciembre de 2018, personal del Departamento de Observación e Investigación Marítima de la Empresa Portuaria Quetzal, colectó muestras de agua para identificar a los organismos presentes. La colecta se realizó a través de un arrastre horizontal con una red para fitoplancton de $20 \mu \mathrm{m}$, durante $5 \mathrm{~min}$, a una velocidad constante de dos nudos, esto con el fin de determinar la biodiversidad de microalgas asociada en al FAN. Al mismo tiempo, se colectaron $500 \mathrm{~mL}$ de muestra superficial de agua, para cuantificar el número de células del dinoflagelado que provocó la decoloración superficial. Las muestras se fijaron con una solución de lugol ácido, como lo describen Cortés-Altamirano, Hernández-Becerril y Luna- Soria (1996), y transportadas para su identificación al Centro de Estudios del Mar y Acuicultura, de la Universidad de San Carlos de Guatemala.

Las muestras colectadas fueron analizadas con microscopio óptico, para el recuento total de los organismos (cel/L) presentes, por medio de cámaras de conteo de Sedgwick Rafter. Para la determinación de los géneros de dinoflagelados, se utilizó el protocolo descrito por Vargas-Montero y Freer (2003), utilizando un 


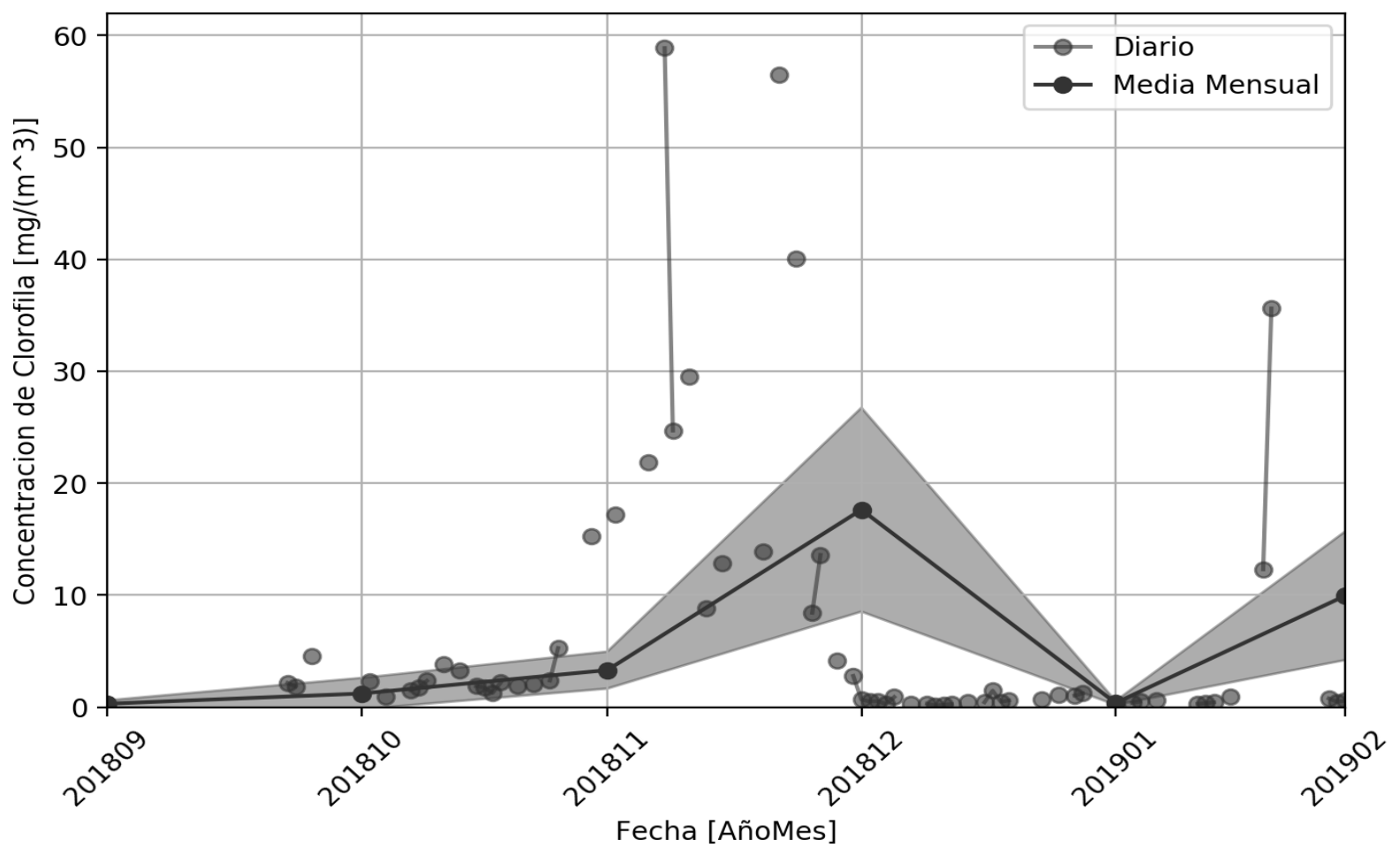

Figura 1. Grafica de la concentración de clorofila en el sitio de Puerto Quetzal, Escuintla, Guatemala, diciembre 2018. Serie temporal de Concentración de Clorofilia frente a Puerto Quetzal instrumento MODIS-Agua (13.90 N, 90.79 O).

microscopio electrónico de barrido (Hitachi, S2360N), propiedad del Centro de Investigación en Estructuras Microscópicas (CIEMic) de la Universidad de Costa Rica.

Conjuntamente, los días 20 y 27 de diciembre de 2018, se recolectaron moluscos bivalvos del género Crassostrea sp., para la cuantificación de saxitoxina, a través del método oficial 959.08.16 AOAC (bioensayo en ratón), en el Laboratorio Nacional de Salud, a través de la cooperación de la Dirección de Normatividad de Pesca y Acuicultura del Ministerio de Agricultura, Ganadería y Alimentación (MAGA).

El FAN consistió en parches de longitud variable entre 50-500 m de largo y aproximadamente $50 \mathrm{~m}$ de ancho, paralelos a la dársena de Puerto Quetzal. Las especies que conformaron la población de dinoflagelados fueron: P. bahamense, Prorocentrum sigmoide, y Dinophysis caudata (Figura 2). En las muestras se observó predominancia de $P$. bahamense, que alcanzó densidades de 3,000 cel/L (Figura 3). P. bahamense presentó una forma semi-redonda poligonal comprimida, tanto en la parte posterior como anterior, con longitud de $50.08(5.30) \mu \mathrm{m}$ y una altura de $60.04(4.05) \mu \mathrm{m}(\mathrm{n}=5)$. La estructura del poro apical presenta prolongación y en la parte de la hipoteca se observan dos espinas, de las cuales la izquierda no es mayor a la espina derecha. Los resultados del bioensayo ratón reflejaron concentraciones de saxitoxina de $8,236 \mathrm{UR} / 100 \mathrm{~g}$ y $6,559 \mathrm{UR} / 100 \mathrm{~g}$, para los días 20 y 27 de diciembre, respectivamente.

\section{Discusión}

El presente caso muestra que los florecimientos de $P$. bahamense poseen un rango de distribución amplio y que siguen proliferando a lo largo de la costa del Pacífico. P. bahamense produce toxinas paralíticas (Gedaria et al., 2007; Hallegraeff \& Maclean, 1989; Usup, Ahmad, Matsuoka, Lim, \& Leaw, 2012), en su mayoría saxitoxina y neosaxitoxina, las cuales contribuyen con el 85-98 \% de la composición total (Gedaria, Luckas, 


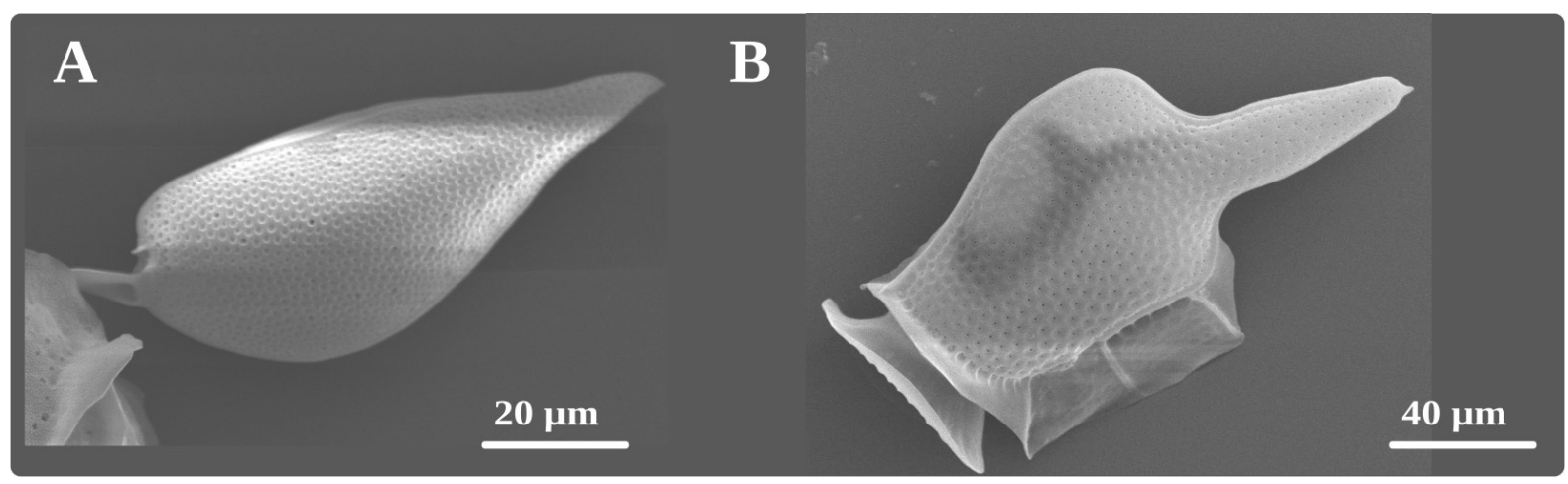

Figura 2. Estructura de fitoplancton planctónico: (A) Prorocentrum sigmoides, y (B) Dinophysis caudata.

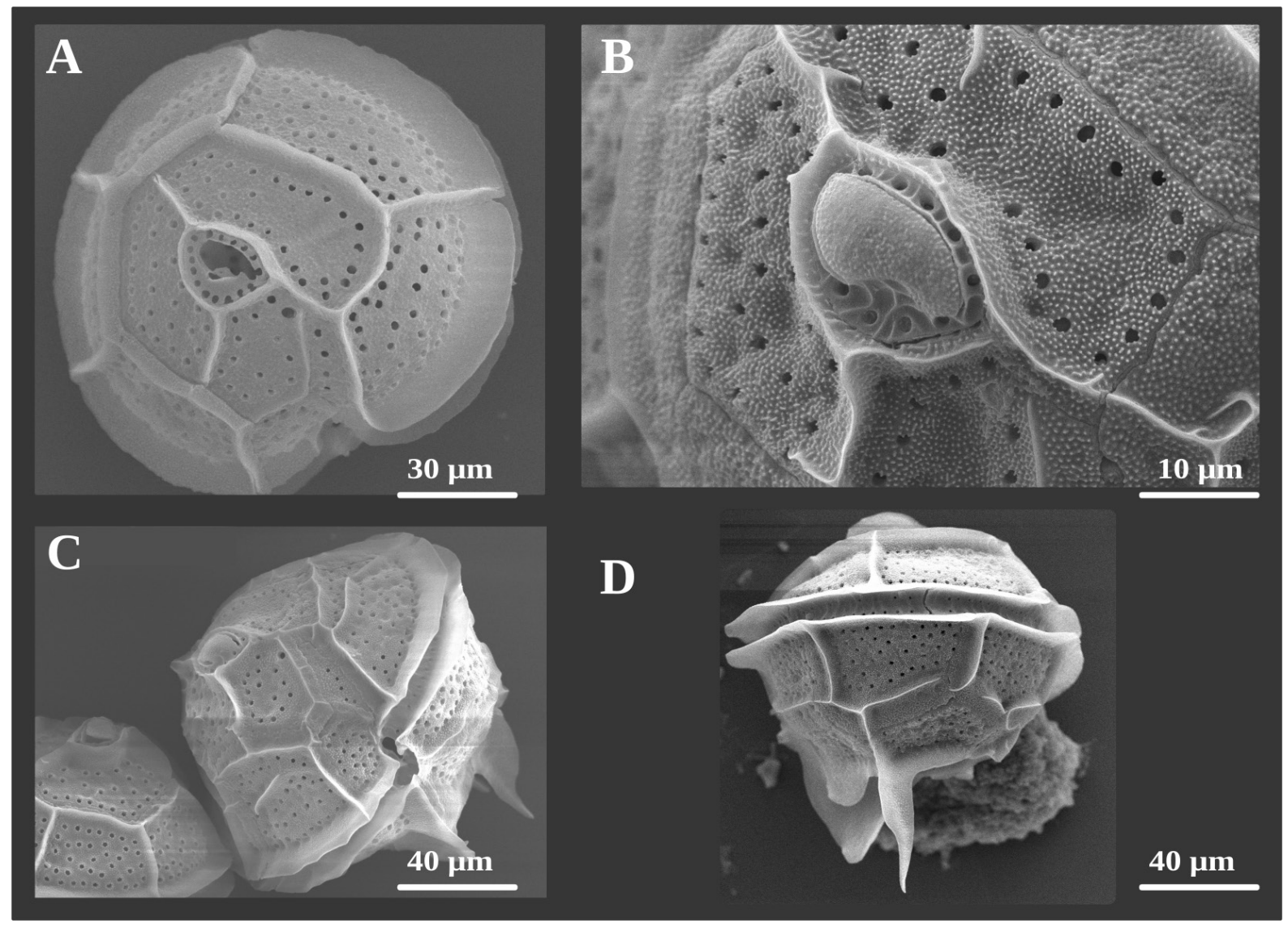

Figura 3. Características morfológicas de Pyrodinium bahamense. Imágenes al microscopio electrónico de barrido, las células presentan una forma semi-redonda poligonal comprimida (A y B) y presentan poro apical con prolongación (C) y dos espinas de las cuales la izquierda no es mayor de la derecha (D). 
Reinhardtb, \& Azanza, 2007). Aunque es difícil predecir un evento de FAN, se conoce que son fenómenos biológicos que ocurren naturalmente como resultados de combinaciones climáticas, hidrobiológicas y por actividades antropogénicas (Berdalet et al., 2011).

Un factor determinante para que ocurran florecimientos algales es la presencia de nutrientes, en particular nitrógeno, el cual estimula la producción primaria de las microalgas (Paerl et al., 2008). El aumento en la concentración de clorofila $a$, y la variación en las condiciones ambientales, como corrientes oceánicas y dirección del viento, facilitan el aumento de producción primaria de microalgas (Van Dolah, 2000) y pueden aumentar el riesgo en la ocurrencia de FAN. Por otra parte, la toxicidad de un FAN dependerá del tipo de toxinas (o químicos producidos) y de las condiciones ambientales (Cabrera, Navarro, \& Altamirano, 1993).

La concentración de saxitoxina registrada en 2018 fue la más alta que se tiene registro en la costa del Pacífico de Guatemala desde el primer FAN reportado (Rosales-Loessener, 1989; Rosales- Loessener et al., 1989). Afortunadamente, el FAN de Pyrodinium en diciembre de 2018 en Guatemala no provocó casos de intoxicación en humanos, según fuente del Ministerio de Salud Pública y Asistencia Social. Sin embargo, la presencia de este tipo de toxina en moluscos bivalvos, es una señal de alarma para la salud humana, por lo cual, los muestreos mensuales en las aguas costeras, para la determinación de toxinas en organismos marinos, y la evaluación de la toxicidad en humanos, son esenciales para la prevención y el bienestar de las comunidades que viven cerca de la costa.

Considerando que los FAN representan una amenaza para la salud humana y para el medio acuático, se recomienda continuar con programas de monitoreo para el manejo adecuado de los recursos marino-costeros y realizar estudios para un mayor entendimiento de tales eventos en la costa de Guatemala. Así mismo es necesario investigar su presencia en diferentes regiones y épocas del año, identificar a las especies potencialmente tóxicas más comunes y determinar la concentración de toxinas correspondientes, a fin de determinar si existen patrones temporales y espaciales y ampliar la información sobre estos eventos.

\section{Agradecimientos}

Se reconoce la cooperación con el Centro de Investigación en Estructuras Microscópicas de la Universidad de Costa Rica en el procesamiento y toma de fotografías al microscopio electrónico de barrido de las muestras, y al Laboratorio Nacional de Salud (LNS) de la Dirección General de Regulación, Vigilancia y Control de la Salud, Ministerio de Salud Pública y Asistencia Social, por la realización del bioensayo en ratón. Se agradece a Guillermo Ambrosio por procesar los datos del satélite de órbita polar y a Elisa Blanda por el apoyo en el proceso de redacción.

\section{Referencias}

Alonso-Rodríguez, R., Mendoza-Amezquita, E., Velásquez-López, S. A., Seim, J. A., \& MartínezRodríguez, V. (2015). Florecimientos algales nocivos producidos por Pyrodinium bahamense en Oaxaca, México (2009-2010). Salud Pública de México, 57(4), 343-351. http://doi.org/10.21149/ spm.v57i4.7578

Band-Schmidt, C. J., Bustillos-Guzmán, J. J., LópezCortes, D. J., Nunez-Vasquez, E., \& HernándezSandoval, F. E. (2011). El estado actual de florecimientos algales nocivos en México. Hidrobiologica, 21(3), 381-413.

Berdalet, E., Fleming, L. E., Gowen, R., Davidson, K., Hess, P., Backer, L., ... Enevoldsen, H. (2011). Marine harmful algal blooms, human health and wellbeing: challenges and opportunities in the 21 st century. Journal of the Marine Biological Association of the United Kingdom, 1, 1-62. https://doi.org/10.1017/S0025315415001733

Cabrera, L. M., Navarro, O., \& Altamirano, R. C. (1993). Envenenamiento paralitico por mariscos (PSP) causado por el dinoflagelado Pyrodinium bahamense var. compressum en la costa sureste de México. Anales del Instituto de Ciencias del Mar y Limnología, 20, 43-54.

Carrillo-Ovalle, H. L. (2009). Fase I: Estudio de los florecimientos algales (mareas rojas), en el Pacifico de Guatemala (Fodecyt No. 31-2007). Guatemala: Secretaria Nacional de Ciencia y Tecnología.

Cortés-Altamirano, R., Hernández-Becerril, D. U., \& Luna- Soria, R. (1996). Red tides in México. In T. Yasumoto, Y. Oshima \& Y. Fukuyo (Eds.), Harmful and toxic algal blooms (pp. 101-104). Paris: IOC/UNESCO. 
Gedaria, A. I., Luckas, B., Reinhardtb, K., \& Azanza, R. V. (2007). Growth response and toxin concentration of cultured Pyrodinium bahamense var. compressum to varying salinity and temperature conditions. Toxicon, 50(4), 518-529. https://doi.org/10.1016/j.toxicon.2007.04.021

Hallegraeff, G., \& Maclean, J. L. (Eds). (1989). Biology, Epidemiology and Management of Pyrodinium Red Tides. Manila, Philippines: International Center for Living Aquatic Resources Management.

Herrera-Sepúlveda, A., Sierra-Beltrán, A., \& Hernández-Saavedra, N. (2008). Floraciones algales nocivas: Perspectivas y estrategias biotecnológicas para su detección. BioTecnologia, 12(1), 23-40.

Paerl, H. W., Joyner, J. J., Joyner, A. R., Arthur, K., Paul, V., O’Neil, J. M., \& Heil, C. A. (2008). Cooccurence of dinoflagellate and cyanobacterial harmful algal blooms in southwest Florida coastal waters: Dual nutrient (N and P) input controls. Marine Ecology Progress Series, 371, 143-153. https://doi.org/10.3354/meps07681

Plate, L. (1906). Pyrodinium bahamense n.g., n. sp. die Leucht-Peridinee des "Feuersees" von Nassau, Bahamas. Archiv für Protistenkunde, 7, 411-442.

Rosales-Loessener, F. (1989). The Guatemalan experience with red tides and paralytic shellfish poisoning. In: G. Hallegraeff, \& J. L., Maclean (Eds). Biology, Epidemiology and Management of Pyrodinium Red Tides (pp. 49-51), Manila, Philippines: International Center for Living Aquatic Resources Management.
Rosales-Loessener, F. M. W. (1989). Toxic shellfish poisoning in Guatemala. En T. Okaichi, D. M. Anderson \& T. Nemoto (Eds,), Red Tides: Biology, Environmental Science and Toxicology (pp. 113-116). Amsterdam: Elsevier Science Publising.

Smayda, T. J. (1997). Harmful algal blooms: Their ecophysiology and general relevance to phytoplankton blooms in the sea. Limnology and Oceanography, 42(5), 1137-1153. https://doi. org/10.4319/1o.1997.42.5_part_2.1137

Usup, G., Ahmad, A., Matsuoka, K., Lim, P., \& Leaw, C. P. (2012). Biology, ecology and Bloom dynamics of the toxic marine dinoflagellate Pyrodinium bahamense. Harmful Algae, 14, 301-312. https:// doi.org/10.1016/j.hal.2011.10.026

Van Dolah, F. M. (2000). Marine algal toxins: Origins, health effect, and their increased occurrence. Environmental Health Perspectives, 108(Supp. 1), 133-141. https://doi.org/10.1289/ehp.00108s1133

Vargas-Montero, M., \& Freer, E. (2003). Co-occurrence of different morphotypes of Pyrodinium bahamense during an extensive bloom in the Gulf of Nicoya, Costa Rica. En A. Villalba, B. Reguera, J. L. Romalde \& R. Beiras (Eds.), Molluscan shellfish safety (pp. 211-217). Vigo, España, París, Francia: Xunta de Galicia and IOCUNESCO. 